\title{
A Theoretical Comparison of Different Target Registration Error Estimators
}

\author{
Mehdi Hedjazi Moghari ${ }^{1}$, Burton $\mathrm{Ma}^{2, \star}$, and Purang Abolmaesumi ${ }^{1,2,3}$ \\ ${ }^{1}$ Department of Electrical and Computer Engineering, Queen's University, Canada \\ ${ }^{2}$ School of Computing, Queen's University, Canada \\ ${ }^{3}$ Department of Surgery, Queen's University, Canada \\ purang@cs . queensu.ca
}

\begin{abstract}
Estimation of target registration error (TRE), a common measure of the registration accuracy, is an important issue in computer assisted surgeries. Within the last decade, several new approaches have been developed to estimate either the mean squared value of TRE or the distribution of TRE under different noise conditions. In this paper, we theoretically demonstrate that all the proposed algorithms converge to a general Maximum Likelihood (ML) solution. Numerical simulations are performed to validate our derivations. Using experimentally measured fiducial localization error, we provide an example of TRE prediction in the presence of anisotropic noise.
\end{abstract}

\section{Introduction}

In the guidance systems designed for computer assisted surgeries (such as hip, spine and neurosurgeries), rigid transformations are mainly used to perform the registration. In these surgeries, the accuracy of the performed registration is an important factor which has direct impact on the operation quality. Due to the noise in medical data sets, there is always error in the performed registrations, and therefore, a measure is required to determine the registration accuracy.

Maurer et al. 1] suggested three useful measures for analyzing the accuracy of the registration: 1) Fiducial localization error (FLE), which is the error in the location of the points or fiducials; 2) Fiducial registration error (FRE), which is the root mean squared distance among corresponding points used in registration; and 3) Target registration error (TRE), which is the distance, after registration, between a pair of corresponding points which are not used in registration. Fitzpatrick and West [2] assumed that FLE (noise in the data set) has an identical and isotropic Gaussian distribution and used a first-order Taylor series approximation to calculate the expected mean squared value of TRE. They were the first to derive a closed-form solution to estimate the mean squared value of TRE, and they used Monte Carlo simulations to approximate the distribution of TRE. In 2006, Moghari and Abolmaesumi [3] derived a general closed-form solution to calculate the distribution of TRE at any target locations using the assumption that FLE has an identical and isotropic distribution. In 2007, Ma et al. 4]

^ The first two authors had equal contributions.

D. Metaxas et al. (Eds.): MICCAI 2008, Part II, LNCS 5242, pp. 1032 1040, 2008.

(C) Springer-Verlag Berlin Heidelberg 2008 
argued that in real applications FLE might be better modeled by identical and anisotropic Gaussian noise. Based on the spatial stiffness theory, they derived a solution which estimates the mean squared value of TRE in the presence of this noise distribution. Seilhorst et al. [5], also in 2007, utilized the transformation of covariance method, proposed by Hoff and Vincent [6], to estimate the distribution of TRE when FLE has an inhomogeneous and anisotropic (heterogeneous) Gaussian distribution. Similarly, Wiles et al. 7, in 2008, modified Fitzpatrick and West algorithm to derive another closed-form solution to estimate the mean squared value of TRE in the presence of identical and anisotropic Gaussian FLE. Their algorithm is based on a least mean squares (LMS) solution to the fiducial registration problem. One can show that their method can be improved by using a weighted LMS algorithm which is a more optimum solution for anisotropic FLE [8]. Most recently, Moghari and Abolmaesumi [8] utilized the Maximum Likelihood (ML) algorithm to estimate the distribution of TRE when FLE has a heterogeneous distribution in the data sets. Furthermore, they demonstrated that the closed-form solution originally proposed by them in [3] is valid not only for identical and isotropic FLE, but also for a heterogenous noise condition.

In this paper, we mathematically show that, when FLE has a heterogenous Gaussian distribution, the spatial stiffness [4] and transform of covariance [5] algorithms both converge to the ML solution [8]. If FLE has an identical and anisotropic Gaussian distribution, the modified Wiles algorithm using weight LMS also converges to the ML solution. Finally, when FLE has an identical and isotropic distribution, all the algorithms (spatial stiffness, transform of covariance, Wiles, and Fitzpatrick and West algorithms) converge to a unique solution. Our derivations are further validated by numerical and experimental simulations.

\section{Method}

In this section, the maximum likelihood [8, spatial stiffness 4, transform of covariance [5], Wiles [7, and Fitzpatrick and West [2] algorithms are briefly explained. Then, it is shown that when FLE has an arbitrary Gaussian distribution, the first three algorithms converge to a unique solution; and when FLE has an identical and isotropic Gaussian distribution, all the algorithms converge to the same solution.

\subsection{Maximum Likelihood Algorithm}

Assume data set $\mathbf{X}$, as a $3 \times N$ matrix ( $N$ is number of points or fiducials), is rigidly registered to data set $\mathbf{Y}$ :

$$
\mathbf{y}_{i}=\mathbf{R} \mathbf{x}_{i}+\mathbf{t}+\mathbf{n}_{i}
$$

where the $i$ th columns of $\mathbf{X}$ and $\mathbf{Y}$, i.e. $\mathbf{x}_{i}$ and $\mathbf{y}_{i}$, represent a pair of corresponding points in the two data sets. The vector $\mathbf{n}_{i}$ is a zero-mean random vector with probability density function $p_{i}$, that models FLE distribution for the $i$ th corresponding point in the data sets $\mathbf{X}$ and $\mathbf{Y} . \mathbf{R}_{(\theta)}$ is a rotation matrix in terms of 
rotation vector $\theta$ consisting of three rotation angles, and $\mathbf{t}$ is a translation vector. Using the likelihood function [8], covariance matrix of the transformation parameters can be calculated as:

$$
\boldsymbol{\Sigma}=\left(\begin{array}{ll}
\boldsymbol{\Sigma}_{\mathbf{t t}} & \boldsymbol{\Sigma}_{\mathbf{t} \theta} \\
\boldsymbol{\Sigma}_{\theta \mathbf{t}} & \boldsymbol{\Sigma}_{\theta \theta}
\end{array}\right)=\left(\begin{array}{ll}
-\mathbf{J}_{\mathrm{tt}} & -\mathbf{J}_{\mathbf{t} \theta} \\
-\mathbf{J}_{\mathbf{t} \theta}^{T} & -\mathbf{J}_{\theta \theta}
\end{array}\right)^{-1}
$$

where $\mathbf{J}_{\mathbf{t} \mathbf{t}}, \mathbf{J}_{\mathbf{t} \theta}$ and $\mathbf{J}_{\theta \theta}$ are the second derivatives of the log-likelihood function $f=\log \prod_{i=1}^{N} p_{i}\left(\mathbf{y}_{i} \mid \mathbf{x}_{i}, \mathbf{t}, \theta\right)$, in terms of $\mathbf{t}$ and $\theta$, respectively. If the probability density function of FLE at the $i$ th point is a zero-mean Gaussian distribution with covariance matrix $\boldsymbol{\Lambda}_{i}$, i.e. $\mathcal{N}\left(0, \boldsymbol{\Lambda}_{i}\right)$, then $\mathbf{J}_{\mathbf{t t}}, \mathbf{J}_{\mathbf{t} \theta}$ and $\mathbf{J}_{\theta \theta}$ can be mathematically calculated as:

$$
\begin{aligned}
& \mathbf{J}_{\mathrm{tt}}=\frac{\partial^{2} f}{\partial \mathbf{t} \partial \mathbf{t}}=-\sum_{i=1}^{N} \boldsymbol{\Lambda}_{i}^{-1}, \\
& \mathbf{J}_{\mathbf{t} \theta}=\frac{\partial^{2} f}{\partial \mathbf{t} \partial \theta}=-\sum_{i=1}^{N} \boldsymbol{\Lambda}_{i}^{-1} \overbrace{\left(\begin{array}{ccc}
0 & x_{i}^{z} & -x_{i}^{y} \\
-x_{i}^{z} & 0 & x_{i}^{x} \\
x_{i}^{y} & -x_{i}^{x} & 0
\end{array}\right)}^{\mathbf{B}_{i}^{T}}, \\
& \mathbf{J}_{\theta \theta}=\frac{\partial^{2} f}{\partial \theta \partial \theta}=-\sum_{i=1}^{N} \overbrace{\left(\begin{array}{ccc}
0 & -x_{i}^{z} & x_{i}^{y} \\
x_{i}^{z} & 0 & -x_{i}^{x} \\
-x_{i}^{y} & x_{i}^{x} & 0
\end{array}\right)}^{\mathbf{B}_{i}} \boldsymbol{\Lambda}_{i}^{-1}\left(\begin{array}{ccc}
0 & -x_{i}^{z} & x_{i}^{y} \\
x_{i}^{z} & 0 & -x_{i}^{x} \\
-x_{i}^{y} & x_{i}^{x} & 0
\end{array}\right)^{T} .
\end{aligned}
$$

Having calculated the covariance matrix of the transformation parameters $\boldsymbol{\Sigma}$ from Equation (2) by using (3), (4), and (5), one can utilize the closed-form solution proposed in [3] to estimate the distribution of TRE at a target point $\mathbf{r}$.

\subsection{Transform of Covariance Method}

Sielhorst et al. [5] employed the transform of covariance algorithm, introduced by Hoff and Vincent [6], to calculate the covariance matrix of the transformation parameters as follows:

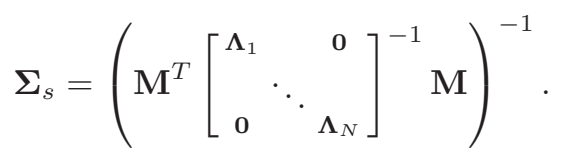

$\mathbf{M}$ is a concatenation of Jacobian matrix of Equation (1) calculated at each point in the data set $\mathbf{X}$ :

$$
\mathbf{M}=\left[\mathbf{J}\left(\mathbf{x}_{1}\right)^{T}, \ldots, \mathbf{J}\left(\mathbf{x}_{N}\right)^{T}\right]^{T}, \quad \mathbf{J}\left(\mathbf{x}_{i}\right)=\left(\begin{array}{cccccc}
1 & 0 & 0 & 0 & x_{i}^{z} & -x_{i}^{y} \\
0 & 1 & 0 & -x_{i}^{z} & 0 & x_{i}^{x} \\
0 & 0 & 1 & x_{i}^{y} & -x_{i}^{x} & 0
\end{array}\right)=\left(\begin{array}{ll}
\mathbf{I} & \mathbf{B}_{i}^{T}
\end{array}\right),
$$

where $\mathbf{I}$ is the identity matrix and $\mathbf{B}_{i}$ is defined in Equation (4). By substituting $\mathbf{J}\left(\mathbf{x}_{i}\right)$ in $\mathbf{M}$, and $\mathbf{M}$ in (6), $\boldsymbol{\Sigma}_{s}$ can be modified to:

$$
\boldsymbol{\Sigma}_{s}=\left(\left(\begin{array}{ccc}
\mathbf{I} & \ldots & \mathbf{I} \\
\mathbf{B}_{1} & \ldots & \mathbf{B}_{N}
\end{array}\right)\left[\begin{array}{ccc}
\boldsymbol{\Lambda}_{1}^{-1} & & \mathbf{0} \\
& \ddots & \\
& & \boldsymbol{\Lambda}_{N}^{-1}
\end{array}\right]\left(\begin{array}{cc}
\mathbf{I} & \mathbf{B}_{1}^{T} \\
\vdots & \vdots \\
\mathbf{0} & \mathbf{B}_{N}^{T}
\end{array}\right)\right)^{-1}=
$$




$$
\left(\left(\begin{array}{ccc}
\mathbf{I} & \ldots & \mathbf{I} \\
\mathbf{B}_{1} & \ldots & \mathbf{B}_{N}
\end{array}\right)\left(\begin{array}{cc}
\boldsymbol{\Lambda}_{1}^{-1} & \boldsymbol{\Lambda}_{1}^{-1} \mathbf{B}_{1}^{T} \\
\vdots & \vdots \\
\boldsymbol{\Lambda}_{N}^{-1} & \boldsymbol{\Lambda}_{N}^{-1} \mathbf{B}_{N}^{T}
\end{array}\right)\right)^{-1}=\left(\begin{array}{ccc}
\sum_{i=1}^{N} \boldsymbol{\Lambda}_{i}^{-1} & & \sum_{i=1}^{N} \boldsymbol{\Lambda}_{i}^{-1} \mathbf{B}_{i}^{T} \\
& \ddots & \\
\sum_{i=1}^{N} \mathbf{B}_{i} \boldsymbol{\Lambda}_{i}^{-1} & & \sum_{i=1}^{N} \mathbf{B}_{i} \boldsymbol{\Lambda}_{i}^{-1} \mathbf{B}_{i}^{T}
\end{array}\right)^{-1} .
$$

Comparing Equations (8) and (2) verifies that both the transform of the covariance and maximum likelihood algorithms reach to the same solution in estimation of the covariance matrix of the transformation parameters. Equation (8) can be used in the closed-form solution derived in 3 to estimate the distribution of TRE at the target location $\mathbf{r}$.

\subsection{Spatial Stiffness Method}

Ma et al. 4, utilized the spatial stiffness theory to estimate the mean squared value of TRE to within a constant factor, when FLE has a zero-mean Gaussian distribution with covariance matrix $\boldsymbol{\Lambda}_{i}=\operatorname{diag}\left(\frac{1}{k_{x_{i}}}, \frac{1}{k_{y_{i}}}, \frac{1}{k_{x_{i}}}\right)$. Using the spatial stiffness theory, the covariance matrix of the transformation parameters is computed as $\boldsymbol{\Sigma}_{b}=\left(\sum_{i=1}^{N} \mathbf{H}_{i}\right)^{-1}$, where

$$
\begin{gathered}
\mathbf{H}_{i}=\left(\begin{array}{cccccc}
k_{x_{i}} & 0 & 0 & 0 & k_{x_{i}} x_{i}^{z} & -k_{x_{i}} x_{i}^{y} \\
0 & k_{y_{i}} & 0 & -k_{y_{i}} x_{i}^{z} & 0 & k_{y_{i}} x_{i}^{x} \\
0 & 0 & k_{z_{i}} & k_{z_{i}} x_{i}^{y} & -k_{z_{i}} x_{i}^{x} & 0 \\
0 & -k_{x_{i}} x_{i}^{z} & k_{x_{i}} x_{i}^{y} & k_{z_{i}}\left(x_{i}^{y}\right)^{2}+k_{y_{i}}\left(x_{i}^{z}\right)^{2} & -k_{z_{i}} x_{i}^{y} x_{i}^{x} & -k_{y_{i}} x_{i}^{x} x_{i}^{z} \\
-k_{y_{i}} x_{i}^{z} & 0 & k_{y_{i}} x_{i}^{x} & -k_{z_{i}} x_{i}^{y} x_{i}^{x} & k_{z_{i}}\left(x_{i}^{x}\right)^{2}+k_{x_{i}}\left(x_{i}^{z}\right)^{2} & -k_{x_{i}} x_{i}^{y} x_{i}^{z} \\
k_{z_{i}} x_{i}^{y} & -k_{z_{i}} x_{i}^{x} & 0 & -k_{y_{i}} x_{i}^{x} x_{i}^{z} & -k_{x_{i}} x_{i}^{y} x_{i}^{z} & k_{y_{i}}\left(x_{i}^{x}\right)^{2}+k_{x_{i}}\left(x_{i}^{y}\right)^{2}
\end{array}\right), \\
\mathbf{H}_{i}=\left(\begin{array}{cc}
\boldsymbol{\Lambda}_{i}^{-1} & \boldsymbol{\Lambda}_{i}^{-1} \mathbf{B}_{i}^{T} \\
\mathbf{B}_{i} \boldsymbol{\Lambda}_{i}^{-1} \mathbf{B}_{i} \boldsymbol{\Lambda}_{i}^{-1} \mathbf{B}_{i}^{T}
\end{array}\right) .
\end{gathered}
$$

By substituting (8) in $\boldsymbol{\Sigma}_{b}$, it can be easily verified that $\boldsymbol{\Sigma}_{b}=\boldsymbol{\Sigma}_{c}=\boldsymbol{\Sigma}$. Having calculated $\boldsymbol{\Sigma}_{b}$, as explained before, one can easily estimate the distribution of TRE at the target location $\mathbf{r}$.

\subsection{Wiles Algorithm}

Wiles et al. 7] derived a closed-form solution that estimates the mean squared value of TRE when FLE has an identical and anisotropic zero-mean Gaussian noise distribution $N\left(0, \boldsymbol{\Lambda}_{i}\right)$. Their algorithm, similar to Fitzpatrick and West algorithm, utilizes a LMS algorithm to estimate the registration parameters and their variances. However, since FLE has an anisotropic distribution, using a weighted LMS leads to a more optimal solution [8]. If the data sets are translated to their origins and aligned along their eigenvectors, and FLE axes are aligned with $X$ and it has an uncorrelated zero mean Gaussian distribution $\left(\boldsymbol{\Lambda}_{i}=\operatorname{diag}\left(\sigma_{x}^{2}, \sigma_{y}^{2}, \sigma_{z}^{2}\right)\right)$, by using a weighted LMS algorithm to calculate the transformation parameters and Wiles et al. algorithm to compute the covariance matrix of the transformation parameters, $\boldsymbol{\Sigma}_{\mathbf{t t}}, \boldsymbol{\Sigma}_{\mathbf{t} \theta}$ and $\boldsymbol{\Sigma}_{\theta \theta}$ can be determined as follows: $\boldsymbol{\Sigma}_{\mathbf{t t}}=\operatorname{diag}\left(\frac{\sigma_{x}^{2}}{N}, \frac{\sigma_{y}^{2}}{N}, \frac{\sigma_{z}^{2}}{N}\right), \boldsymbol{\Sigma}_{\mathbf{t} \theta}=\mathbf{0}$, and 


$$
\boldsymbol{\Sigma}_{\theta \theta}=\operatorname{diag}\left(\frac{1}{\sum_{i=1}^{N}\left(\frac{x_{i}^{z}}{\sigma_{y}}\right)^{2}+\left(\frac{x_{i}^{y}}{\sigma_{z}}\right)^{2}}, \frac{1}{\sum_{i=1}^{N}\left(\frac{x_{i}^{z}}{\sigma_{x}}\right)^{2}+\left(\frac{x_{i}^{x}}{\sigma_{z}}\right)^{2}}, \frac{1}{\sum_{i=1}^{N}\left(\frac{x_{i}^{y}}{\sigma_{x}}\right)^{2}+\left(\frac{x_{i}^{x}}{\sigma_{y}}\right)^{2}}\right),
$$

which match the ones computed from (2), (3), (4), and (5).

\subsection{Fitzpatrick and West Algorithm}

Fitzpatrick and West 2 presented a close-form solution to estimate the mean squared value of TRE when FLE has an isotropic and identical zero-mean Gaussian distribution with covariance matrix $\boldsymbol{\Lambda}_{i}=\sigma^{2} \mathbf{I}$. By using these assumptions that the centroid of the data sets is at the origin and the data sets are aligned along their orthogonal eigenvectors, (3), (4) and (5), and hence, the covariance matrix of the transformation parameters can be calculated as: $\boldsymbol{\Sigma}_{\mathbf{t t}}=\frac{\sigma^{2}}{N} \mathbf{I}$, $\boldsymbol{\Sigma}_{\mathbf{t} \theta}=\mathbf{0}$ and

$\boldsymbol{\Sigma}_{\theta \theta}=\operatorname{diag}\left(\frac{\sigma^{2}}{\sum_{i=1}^{N}\left(x_{i}^{z}\right)^{2}+\left(x_{i}^{y}\right)^{2}}, \frac{\sigma^{2}}{\sum_{i=1}^{N}\left(x_{i}^{z}\right)^{2}+\left(x_{i}^{x}\right)^{2}}, \frac{\sigma^{2}}{\sum_{i=1}^{N}\left(x_{i}^{y}\right)^{2}+\left(x_{i}^{x}\right)^{2}}\right)$,

which match the ones computed by Fitzpatrick and West (i.e. Equations (33), (38) and (39) in [2]).

\section{Results}

In order to test and verify our derivations, numerical and experimental simulations are performed to compare different algorithms' performances in estimating the distribution of TRE at a target location $\mathbf{r}$.

\subsection{Simulation Results}

To perform the simulation, number of points in the data sets is assumed to be 10. Data set $\mathbf{X}$ is generated by drawing 10 fiducial points uniformly within a cube with the sides of $\pm 100 \mathrm{~mm}$. Also, $\mathbf{r}$, the target location, is selected randomly from a cube of sides $\pm 200 \mathrm{~mm}$. For each data set $\mathbf{X}$, we generated two data sets $\mathbf{Y}_{1}$ and $\mathbf{Y}_{2}$ contaminated by isotropic, identical noise and heterogeneous noise, respectively, by perturbing independently the $x, y$ and $z$ components of each point in $\mathbf{X}$ by a zero-mean Gaussian random variable, which models FLE along each orthogonal axis. The distribution of FLE is considered to be $\mathcal{N}\left(0, \sigma^{2} \mathbf{I}\right)$, and $\mathcal{N}\left(0, \operatorname{diag}\left(\sigma_{x}^{2}, \sigma_{y}^{2}, \sigma_{z}^{2}\right)\right)$ for the isotropic and heterogeneous cases, respectively. $\sigma^{2}$ is assumed to be $10 \mathrm{~mm}^{2}$ and $\sigma_{x}^{2}, \sigma_{y}^{2}$, and $\sigma_{z}^{2}$ are randomly chosen from a uniform distribution with the side of $0 \mathrm{~mm}^{2}$ and $20 \mathrm{~mm}^{2}$. In this way, we assume FLE has 
an identical isotropic zero-mean Gaussian distribution and heterogeneous zeromean Gaussian distribution for each point in the data set, respectively. Then, $\mathbf{X}$ is registered to $\mathbf{Y}_{1}$ and $\mathbf{Y}_{2}$, and TRE is measured at $\mathbf{r}$.

The distribution of TRE is numerically estimated by using repeated perturbation and registration steps 100, 000 times (Monte Carlo simulations). Also, the maximum likelihood [8], transform of covariance [5], spatial stiffness 44, Wiles [7] and Fitzpatrick and West 2 algorithms are used to compute the distribution of TRE. Figures 1 and 2 display the computed probability density functions of TRE at $\mathbf{r}=[184,91,-35]^{T}$ using numerical simulations and the other algorithms when FLE has an isotropic and identical, and a heterogenous zero-mean Gaussian distribution, respectively.

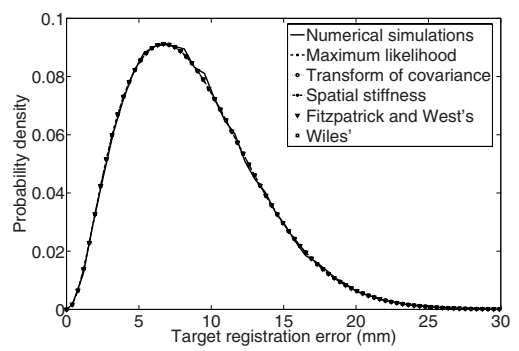

Fig. 1. Probability density of TRE at $\mathbf{r}$, when FLE has isotropic and identical zero-mean Gaussian noise

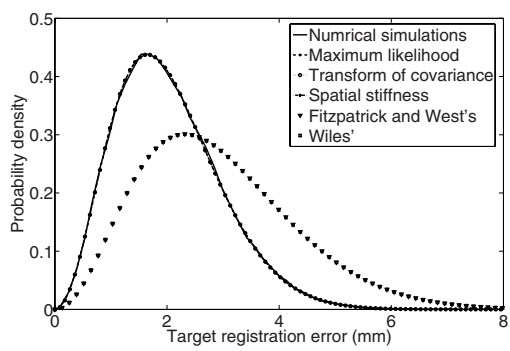

Fig. 2. Probability density of TRE at $\mathbf{r}$, when FLE has heterogeneous zero-mean Gaussian noise

\subsection{Experimental Results}

The TRE estimators require that the FLE magnitude (Fitzpatrick and West algorithm) or the FLE covariance be specified. We attempted to characterize the FLE of an optical tracking system by measuring the FRE; the FRE is known to approach the FLE as the number of fiducials increases 9]. We used an Optotrak Certus (Northern Digital Inc., Waterloo, Ontario, Canada) for our optical tracking system. A large coordinate reference frame (CRF) was constructed by mounting 20 infrared light emitting diodes (IREDs) on the perimeter of a square of size $380 \times 380 \mathrm{~mm}$ on a rigid board. The board was mounted vertically on an optical bench such that the board could rotate about its vertical axis. The optical bench was positioned well inside the working volume of the Certus.

We acquired sets of measurements of the CRFs at 50 different locations on a horizontal plane inside the calibrated working volume of the Certus. For each set of measurements, we rotated the CRFs from -50 to +50 degrees in 10 degree increments about the vertical axis. At each angular increment, the CRF was kept stationary relative to the Certus and 200 measurements of the 3D IRED locations were acquired over a period of 2 seconds; each IRED location was taken to be the average location (over the 200 measurements). Note that this process of measuring the location of a stationary IRED does not yield the FLE; 

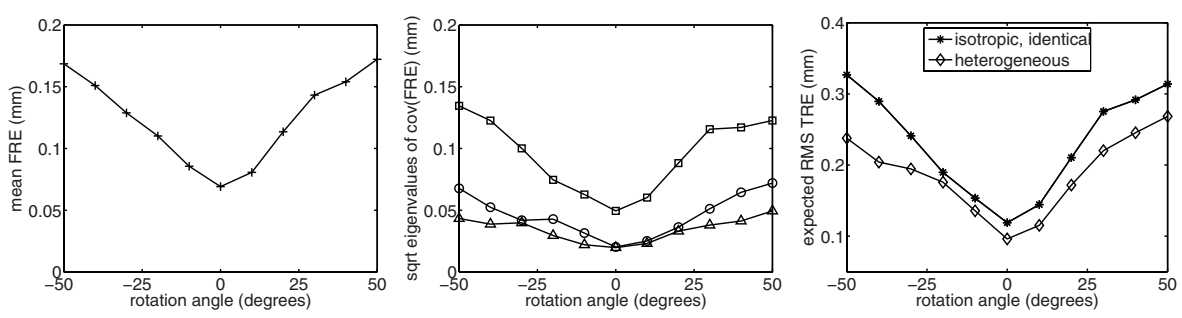

Fig. 3. (Left) Experimentally determined FRE magnitude. (Middle) Principal components of FRE covariance. (Right) Theoretical predictions of RMS TRE using the experimentally determined FLE.

it produces a measurement of the jitter [10, which is only one component of FLE. The Certus was not moved throughout the entire experiment.

For each measurement $P$ of the 20 IREDS taken at 0 degrees rotation, we registered all sets of measurements taken throughout the working volume to $P$ and computed the vectorial FRE for each IRED; the registrations were performed using Horn's method [11. The root mean square (RMS) of the empirical FRE magnitude and the square root of the eigenvalues of the covariance of FRE are shown in Figure 3. We observed that the covariance matrix of FRE was diagonally dominant for rotation angles up to \pm 20 degrees, but the covariances (off-diagonal elements) became significant for larger rotation angles. Using the estimated FLE covariances, we computed the expected RMS TRE using the estimators we have studied [24578] for a CRF used in a previous publication [4]. For Fitzpatrick and West's algorithm we used the root means square magnitude of the experimentally determined FLE. The CRF was a tetrahedral configuration of 4 fiducial markers. The results, shown in Figure 3 , indicate that the best case TRE occurs when the CRFs directly face the tracking cameras, a trend opposite to that reported in [4] where a simple constant anisotropic noise model was used. For this particular CRF, assuming isotropic, identical noise results in an overestimated value of TRE.

\section{Discussion}

Using isotropic and identical zero-mean Gaussian noise for FLE, our numerical simulation results, as expected and shown in Figure 1, verify that all the algorithms estimate the same distribution of TRE at the target location $\mathbf{r}$ as the one obtained by Monte Carlo simulations. However, when FLE has a heterogeneous zero-mean Gaussian distribution, as shown in Figure 2, only transform of covariance and spatial stiffness algorithms converge to the ML solution that closely follows the result obtained from Monte Carlo simulations. The same observation is made in the performed experimental simulations discussed in the remainder.

Using a simple anisotropic noise model for FLE, where the noise component magnitudes were constant, a theoretical model of TRE were shown to predict a 
peak in TRE when the CRF directly faces the camera (equivalent to our 0 degrees of rotation) 4. Using an empirical model of FLE, we have shown the exact opposite: expected TRE is minimized when the CRF directly faces the camera. The reason for this is that the FLE noise magnitude and covariance appear to depend on the orientation of the IREDs relative to the tracking camera; this angular dependence has been observed by other researchers [12]13 but we were unable to find quantitative values to use in our models.

The theoretical models of TRE appear to be accurate when tested using simulations but their predictions can only be as good as the noise models they use. This begs the question: how do we construct good noise models? We attempted to experimentally measure FLE and its distribution and used the estimated covariances in the TRE models. Other work that modelled measurement error starting at the image plane of the cameras has recently been reported [514]. Given accurate noise models, we can begin to explore whether or not methods that account for heteroscedastic noise significantly outperform least-squares methods for real, high-accuracy tracking systems.

\section{Conclusion}

For the first time, we unified all the algorithms proposed to estimate the distribution of TRE at a target location $\mathbf{r}$. It is theoretically and experimentally shown that if FLE has inhomogeneous and anisotropic zero-mean Gaussian noise, then the transform of covariance and spatial stiffness algorithms converge to the Maximum Likelihood solution. Also, if FLE has an identical and anisotropic distribution, a modified Wiles algorithm also converges to the ML solution. Finally, all algorithms including Fitzpatrick and West derive the same distribution of TRE at a target location when FLE has identical and isotropic zero-mean Gaussian noise. In real applications, noise might not have a Gaussian distribution, and that would be beneficial to compare these algorithms for the case where FLE has a distribution which is not necessarily Gaussian.

\section{References}

1. Maurer, C.R., McCrory, J.J., Fitzpatrick, J.M.: Estimation of accuracy in localizing externally attached markers in multimodal volume head images. In: SPIE, vol. 1898, pp. 43-54 (1993)

2. Fitzpatrick, J.M., West, J.B.: The distribution of target registration error in rigidbody point-based registration. IEEE TMI 20(9), 917-927 (2001)

3. Moghari, M.H., Abolmaesumi, P.: A high-order solution for the distribution of target registration error in rigid-body point-based registration. In: Larsen, R., Nielsen, M., Sporring, J. (eds.) MICCAI 2006. LNCS, vol. 4191, pp. 603-611. Springer, Heidelberg (2006)

4. Ma, B., Moghari, M.H., Ellis, R.E., Abolmaesumi, P.: On fiducial target registration error in the presence of anisotropic noise. In: Ayache, N., Ourselin, S., Maeder, A. (eds.) MICCAI 2007, Part II. LNCS, vol. 4792, pp. 628-635. Springer, Heidelberg (2007) 
5. Sielhorst, T., Bauer, M., Wenisch, O., Klinker, G., Navab, N.: Online estimation of the target registration error for n-ocular optical tracking systems. In: Ayache, N., Ourselin, S., Maeder, A. (eds.) MICCAI 2007, Part II. LNCS, vol. 4792, pp. 652-659. Springer, Heidelberg (2007)

6. Hoff, W., Vincent, T.: Analysis of head pose accuracy in augmented reality. IEEE Trans. Vis. Comput. Graph. 6(4), 319-334 (2000)

7. Wiles, A.D., Likholyot, A., Frantz, D.D., Peters, T.M.: A statistical model for point-based target registration error with anisotropic fiducial localizer error. IEEE TMI 27(3), 378-391 (2008)

8. Moghari, M.H., Abolmaesumi, P.: Maximum likelihood estimation of the distribution of target registration error. In: SPIE, vol. 6918, pp. 1-12 (2008)

9. Fitzpatrick, J.M., West, J.B., Maurer Jr., C.R.: Predicting error in rigid-body point-based registration. IEEE TMI 17(5), 694-702 (1998)

10. Khadem, R., Yeh, C.C., Sadeghi-Tehrani, M., Bax, M.R., Johnson, J.A., Welch, J.N., Wilkinson, E.P., Shahidi, R.: Comparative tracking error analysis of five different optical track ing systems. Computer Aided Surgery 5, 98-107 (2000)

11. Horn, B.K.P.: Closed-form solution of absolute orientation using unit quaternions. J. Opt. Soc. Am. A 4, 629-642 (1987)

12. West, J.B., Maurer Jr., C.R.: Designing optically tracked instruments for imageguided surgery. IEEE TMI 23(5), 533-545 (2004)

13. Schmerber, S., Chassat, F.: Accuracy evaluation of a CAS system: Laboratory protocol and results with $6 \mathrm{D}$ localizers, and clinical experiences in otorhinolaryngology. CAS 6(1), 1-13 (2001)

14. Bauer, M., Schlegel, M., Pustka, D., Navab, N., Klinker, G.: Predicting and estimating the accuracy n-ocular optical tracking systems. In: IEEE and ACM International Symposium on Mixed and Augmented Reality, pp. 43-51 (2006) 